\title{
Damage Localization and Quantification of Truss Structure Based on Electromechanical Impedance Technique and Neural Network
}

\author{
Cunfu He, Shen Yang, Zenghua Liu, and Bin Wu \\ College of Mechanical Engineering and Applied Electronics Technology, Beijing University of Technology, Pingleyuan 100, \\ Chaoyang District, Beijing 100124, China
}

Correspondence should be addressed to Cunfu He; hecunfu@bjut.edu.cn

Received 18 December 2013; Revised 26 May 2014; Accepted 5 June 2014; Published 29 June 2014

Academic Editor: Gyuhae Park

Copyright (C) 2014 Cunfu He et al. This is an open access article distributed under the Creative Commons Attribution License, which permits unrestricted use, distribution, and reproduction in any medium, provided the original work is properly cited.

\begin{abstract}
Truss structure is widely used in civil engineering. However, it is difficult to quantitatively monitor the state of truss structures because of the connection diversity and complexity of truss structures. In this paper, electromechanical impedance (EMI) technique was proposed to measure impedance spectra by using PZT elements and backpropagation (BP) neural network was used as an effective nonlinear conversion tool to quantify the health state of truss structures. Firstly, frequency band of the spectrum was experimentally determined by the trial-and-error approach. Then four connection rods of this truss structure were selected for experimental research. These connection rods were loosened gradually with a small angle increment and the impedance spectra were recorded. Then, the measured data were compressed through dividing the frequency range into multiple subbands. And RMSD values of these bands showed that data points were reduced while damage features remained. Finally, one four-layered BP neural network model was constructed based on these compressed data. The research results showed that compressed impedance data could retain their damage features. After the training, the developed neural network model could not only determine the location of loosened rod, but also quantify the loosening levels.
\end{abstract}

\section{Introduction}

As an effective structural health monitoring technique, electromechanical impedance (EMI) technique has gained widespread attention for its traits of high local sensitivity, easy installation of sensors, and nonparametric model analysis [1-3]. EMI technique has unique advantages, especially in the field of real-time health monitoring of some complex and irregular structures, such as truss structures [4] and connection bolts [5]. The influence of the added mass and shape of PZT patches was also reported [6].

Sun et al. [4] first proposed EMI technique to monitor the health state of truss structures and validated the effectiveness and potential of this technique to characterize such complex and irregular structures. Yan et al. [7] combined EMI technique with reverberation matrix method to quantitatively evaluate structural damage in Timoshenko beams. To solve the difficulty in the evaluation of damage severity and location, Yang and Divsholi [8] and Divsholi and Yang [9] divided the whole testing frequency range into multiple subbands. Annamdas and Yang [10] adopted EMI technique to monitor the health state of excavation support structures after experimental study. The result showed that, although the variations in signatures were not exactly proportional to load magnitudes, such unexpected things could be predicted on the site in the presence of loads. These researches proved that the structure state, especially the state of complex and irregular structures, could be expressed with EMI technique. However, it is always difficult to obtain visual results through direct observations of impedance spectrum. It is required to process raw data with artificial intelligence algorithms, such as neural network technology.

Artificial neural network is a kind of intelligent damage identification system. With nonlinear mapping ability, parallel computation ability, and good robustness, the neural network can be used as the postprocessing tool of EMI 


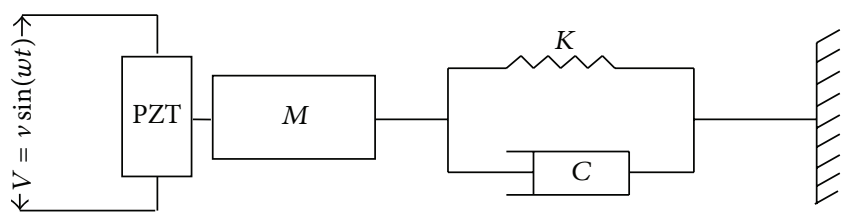

FIGURE 1: One-dimensional model of EMI method.

technique. More related researches already proved that, when monitoring regular structures, this combination of artificial neural network and EMI technique could not only find the existence of defects but also determine the damage conditions $[11,12]$. And Lopes et al. [13] adopted this combination to monitor the health state of irregular structures, evaluate the number of loosened bolts in a steel bridge section, and locate the loosened elements in a space truss structure. However, it is important to locate connection rods and quantify the loosening levels of connection rods for truss structures. In order to quantify the health state of irregular structures, more training samples are required, indicating that neural network has low training rate and indicating the convergence difficulty of the training error. Therefore, it is difficult to evaluate the health states of structures quantitatively, such as truss structure.

In this study, one three-layer truss structure consisting of rods and spherical ball joints was treated as the experimental subject. Circular PZT elements were bonded onto the surface of ball joints to measure impedance spectra and the statistical algorithm was used to process raw data. Firstly, the frequency band of impedance spectra, which were the most sensitive to the variation of structure, were experimentally determined by the trial-and-error approach. Then, when the health state of truss structure was changed, impedance spectra were recorded in this frequency band for the study of damage states. In order to evaluate its health states by using neural network, the statistical algorithm and frequency subdivision method were combined together to preprocess the measured data as input vectors. The dimensions of input vectors can be reduced so that the training rate of neural network can be accelerated while different damage features remained. Finally, BP neural network model was constructed to locate different rods of the truss structure and quantify the loosening levels.

\section{Principle of Structural Health Monitoring Based on EMI Technique}

Theoretical basis of EMI technique was first put forward by Liang et al. [14]. He analyzed one-dimensional model of the structure with PZT element. PZT impedance values were only related to structural mechanical impedance. Figure 1 is the $1 \mathrm{D}$ model adopted by Liang et al. [14].

According to the coupling effect, the positive and inverse piezoelectric effects, and frequency dependent admittance of PZT, $Y(\omega)$ can be expressed as in the following equation:

$$
Y(\omega)=\frac{I}{V}=i \omega a\left(\bar{\varepsilon}_{33}^{T}(1-i \delta)-\frac{Z_{s}(\omega)}{Z_{s}(\omega)+Z_{a}(\omega)} d_{3 x}^{2} \bar{Y}_{x x}^{E}\right),
$$

where $Z_{a}$ is mechanical impedance of PZT; $Z_{s}$ is structural mechanical impedance; $\bar{Y}_{x x}^{E}$ is the complex Young modulus of PZT; $d_{3 x}$ is coupling coefficient between PZT and structure; $\bar{\varepsilon}_{33}^{T}$ is the dielectric constant; $\delta$ is the dielectric loss tangent of the PZT; $a$ is the geometry constant of PZT. According to (1), the first part of the equation only contains parameters of PZT, which remain unchanged if PZT elements are fixed on the surface of structure. Admittance value will be decided only by $Z_{s}$. Thus, structural damages, defects, connection conditions, or other physical changes can be represented by the variation of impedance spectra, which are measured by PZT element.

\section{Experimental Study}

In this paper, one three-layer truss structure was adopted as an experimental subject. For this truss structure sample, total height was $1500 \mathrm{~mm}$ and both length and width were $500 \mathrm{~mm}$. The length of its diagonal rods was $700 \mathrm{~mm}$, and all rods were connected through spherical ball joints with bolt holes. Structural health state was changed when connection rods were loose. The real part of impedance was recorded by PZT elements through a precise impedance analyzer. After compressing and normalization, these data were treated as input samples and neural network was established with these samples. Finally, damage states, that is, loosening levels of connection rods, will be quantified with the trained neural network.

As shown in Figure 2(b), experimental device consists of a precise impedance analyzer (Agilent 4294A), a personal computer (PC), and a truss structure. As experimental subjects, the rods $1,2,3$, and 4 and joints $A$ and $B$ were labeled and illustrated in Figure 2(a). In this study, two identical circular PZT elements, with the $12 \mathrm{~mm}$ diameter and $1 \mathrm{~mm}$ thickness, were bonded on the surface of joints A and B, respectively. When damage states (i.e., loosening levels) of rods 1, 2, 3, and 4 were changed, impedance spectra would be correspondingly tested and recorded. As illustrated in Figure 2(b), in order to quantify the loosening levels of connection rods in experiments, the dial and the pointer were bonded on rod 3 and on the connector between the rod and joint B, respectively. The maximum deflection position of connection rods is 20 degrees from initial position by 2-degree angle increment. Measured impedance data measured with PZT elements can be imported from impedance analyzer to PC through GPIB interface.

3.1. Selection of Experimental Frequency Band. Generally, the most sensitive frequency band is always in the testing frequency range containing multiple peaks $[15,16]$. However, monitoring area will greatly decrease if EMI technique is applied in the excessively high frequency range (above $500 \mathrm{kHz}$ ) [17]. In this paper, the frequency range from $100 \mathrm{kHz}$ to $300 \mathrm{kHz}$ was selected as the initial testing range. Figure 3 shows the real impedance spectra in the range of $100-300 \mathrm{kHz}$ measured at joints A and B under health states. According to Figure 3, it can be found that the peaks of impedance spectra obtained at joints A and B are mainly between $160 \mathrm{kHz}$ and $240 \mathrm{kHz}$. 


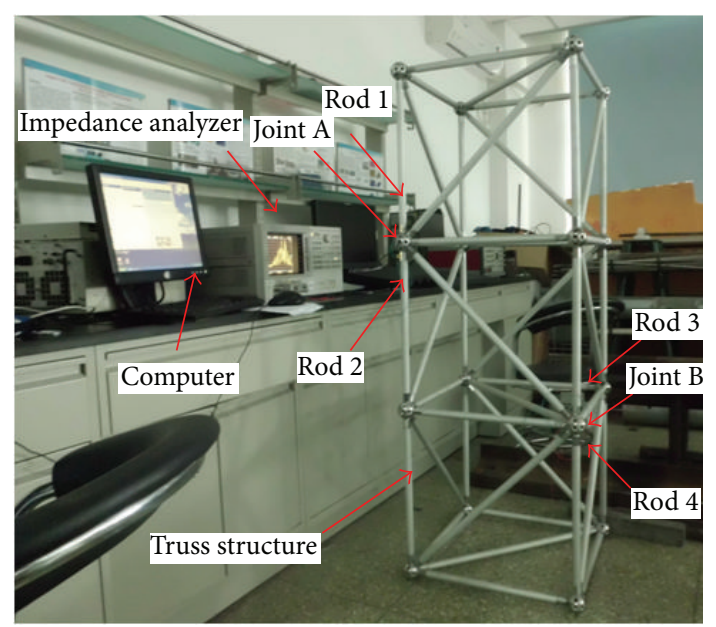

(a)

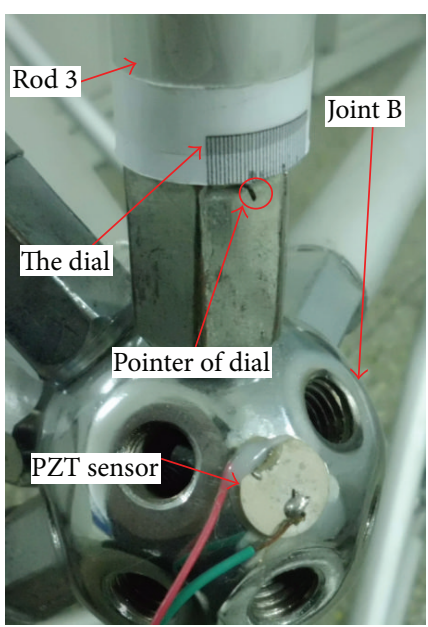

(b)

FIGURE 2: Experimental device and the arrangement of dial and pointer.

Since it is difficult to obtain intuitive results through direct observations of impedance variation, statistical algorithms are usually used to process impedance spectra [1820]. One of the most common statistical algorithms is root mean square deviation (RMSD). Deviation degree among impedance spectra under different damage states can be converted into damage indices with RMSD to evaluate structural damage state according to the comparison results of damage indices.

Since sensitive frequency band could improve the sensitivity of defect detection and quantification, the first step was to determine a proper frequency band by the trial-and-error approach.

Experimental procedure of determining sensitive subbands was shown as follows. Firstly, the whole test frequency range was divided into 10 subbands and impedance spectra were recorded. Each subband was $20 \mathrm{kHz}$ and contained 401 data points. Secondly, rod 1 was loosened to the maximum deflection position (20 degrees) and impedance spectra were recorded as step 1. Then, rod 1 was restored to its initial position. Finally, rod 2 was loosened to the maximum deflection position (20 degrees) and impedance spectra were recorded as step 1.

The whole testing frequency range was divided into 10 subbands and each subband included 401 data points. One RMSD value in each subband can be calculated. Therefore, the total number of RMSD sets was 10 in the whole frequency spectrum. Each RMSD in subband was

$$
M=\sum_{i=1}^{n} \sqrt{\frac{\left(Z_{i, 1}-Z_{i, 2}\right)^{2}}{\left(Z_{i, 1}\right)^{2}}},
$$

where $M$ represents the RMSD value of subband; $Z_{i, 1}$ is the impedance value of the $i$ th frequency point in this subband under health state or baseline state; $Z_{i, 2}$ is impedance value of the $i$ th frequency point in this subband under damage state. It can be found that damage indices will increase with the increment of impedance spectra deviation degree between damage state and health state, indicating the more severe damage state [19].

Firstly, the data obtained from step 1 were treated as baseline state and the data obtained from step 2 were treated as the damage state. Damage indices of different frequency ranges could be calculated when rod 1 was in the loose state. Then, rod 1 was restored to its initial position. The data from step 4 were treated as the damage state while spectrum data from step 1 were treated as baseline. Damage indices of different frequency range could be calculated when rod 2 was in the loose state. Then, joint B was tested according to the same method. Therefore the sensitivity of different subbands was represented by RMSD values, as shown in Figure 4. Series number of bands, such as 1 and 2, represents the subbands of $100-120 \mathrm{kHz}$ and $120-140 \mathrm{kHz}$, respectively. When the monitoring point is joint $\mathrm{A}$, EMI technique shows the highest sensitivity to the loosening levels of rods in the frequency range of $160-220 \mathrm{kHz}$; when the monitoring point is joint $\mathrm{B}$, EMI technique shows the highest sensitivity in the frequency ranges of $120-180 \mathrm{kHz}, 200-220 \mathrm{kHz}$, and $260-300 \mathrm{kHz}$. Therefore, the frequency range of $160-180 \mathrm{kHz}$ was selected for further study, which was very sensitive to the variation of joints $A$ and $B$.

3.2. Tests in Different Loosening Levels of Rods. In order to obtain impedance spectra of the truss structure with different rods under different loosening levels, the experiments were performed according to the following procedure. Firstly, rod 1 was loosened to the maximum deflection position. Then it was gradually tightened in the 2-degree angle increment. Real part of impedance spectrum was recorded after each variation until rod 1 was tightened to its initial state. Each data set contains 401 data points. While the rod was loosened to the maximum deflection position, it was defined as state 1 and the initial position was defined as state 11 . Because impedance spectra corresponding to rod 1 were nearly unchanged, rod 1 was loosened to exceed the maximum deflection position. These spectra could indicate all loosening levels of rod 1 if 


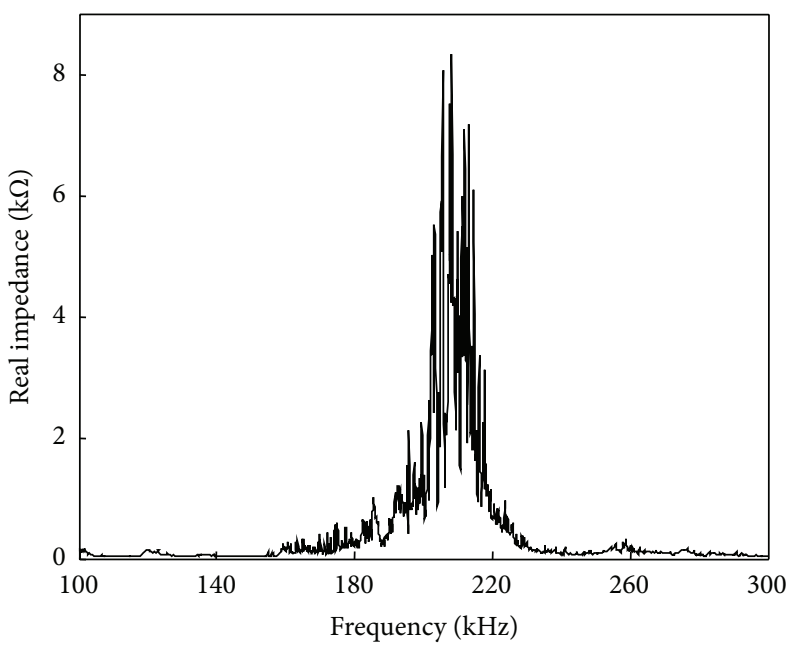

(a)

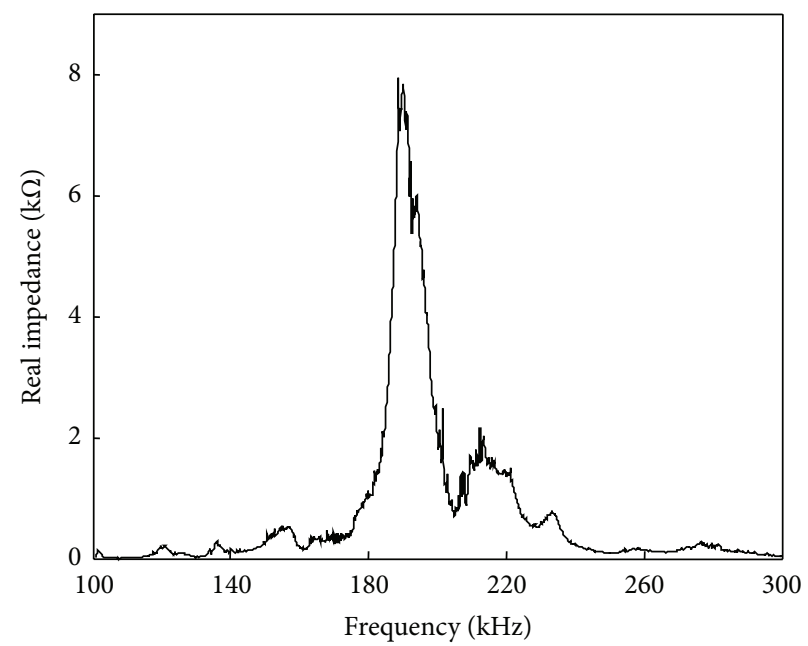

(b)

FIGURE 3: Real impedance spectra in the range of 100-300 kHz measured from (a) joint A and (b) joint $\mathrm{B}$ under health states.

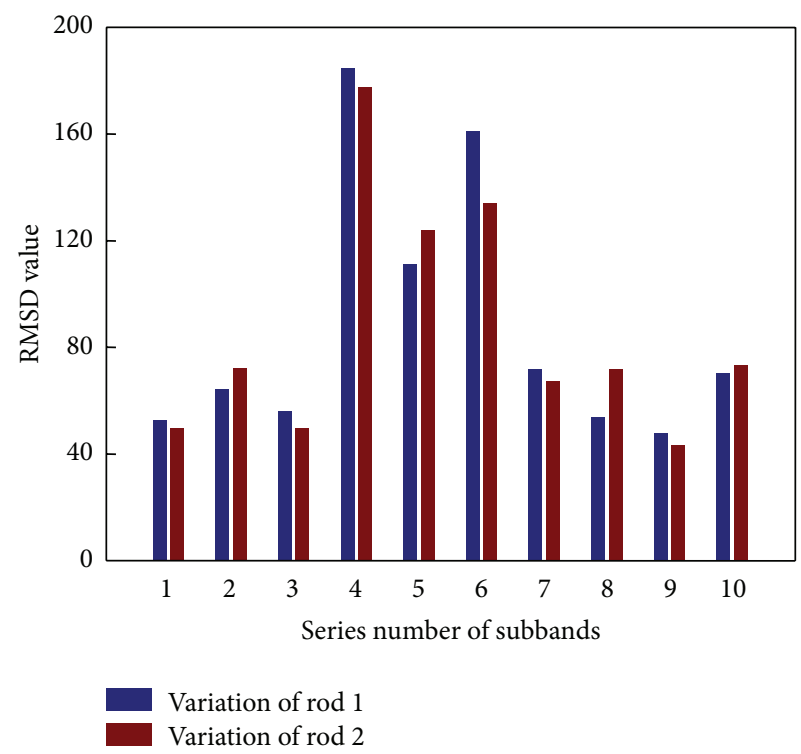

(a)

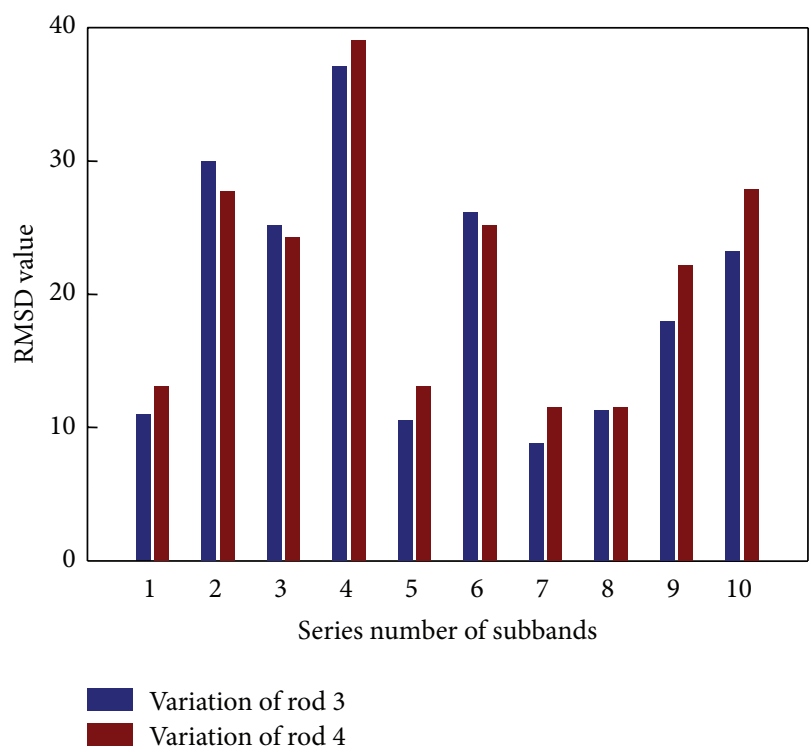

(b)

Figure 4: RMSD values calculated from different subbands when (a) rod 1 and rod 2 and (b) rod 3 and rod 4 of the truss structure were loosened to determine sensitive frequency band.

other rods remained unchanged. The same procedures were performed to determine other damage states. Similarly, after rod 1 returned to its initial position, the spectra of rod 2, rod 3 , and rod 4 were tested according to the same experimental procedure for rod 1 . The total number of data sets was 44 . Figure 5 shows the impedance spectra measured by PZT elements. For the purpose of observation, only the spectra corresponding to the states $1,3,5,7$, and 9 were given, and some parts of these figures were zoomed so that variances of spectra could be found.

From these zoomed parts shown in Figure 5, it is obvious that impedance peaks start to move horizontally and vertically when these rods are tightened gradually. Comparing
Figures 5(a) and 5(b) with Figures 5(c) and 5(d), the most obvious phenomenon is that the spectra measured at joint $\mathrm{A}$ contain more impedance peaks.

In this study, impedance spectra remained nearly unchanged when the looseness of rods was more serious than that of state 1 . Therefore, the spectra corresponding to state 1 of each rod were selected as baseline and RMSD values were calculated with (2). The results were shown in Figure 6. It is compatible with the previous arguments that RMSD values corresponding to joint A are generally larger than those corresponding to joint $\mathrm{B}$, since there are more impedance peaks in spectra measured from joint A. It can be also found that RMSD values corresponding 


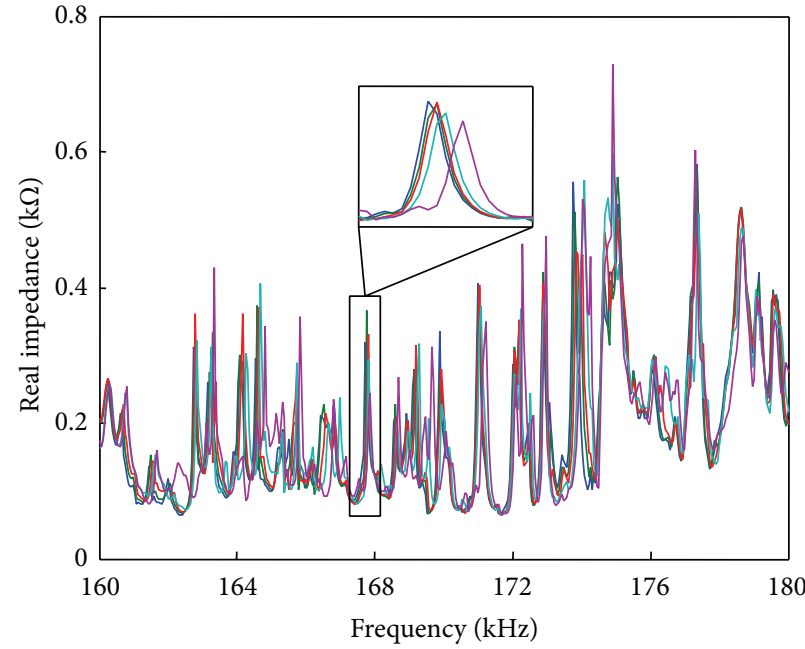

(a)

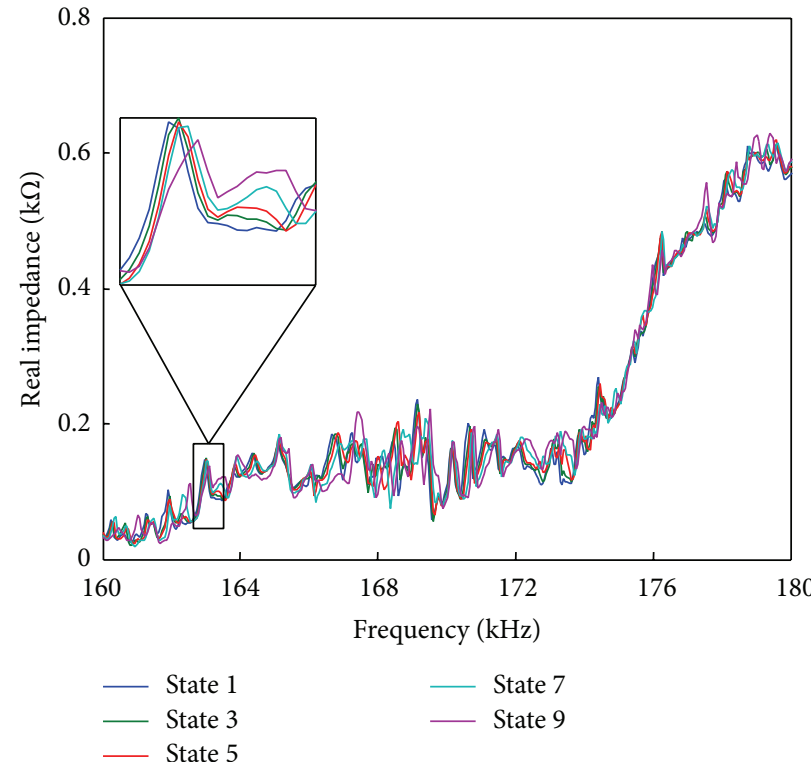

(c)

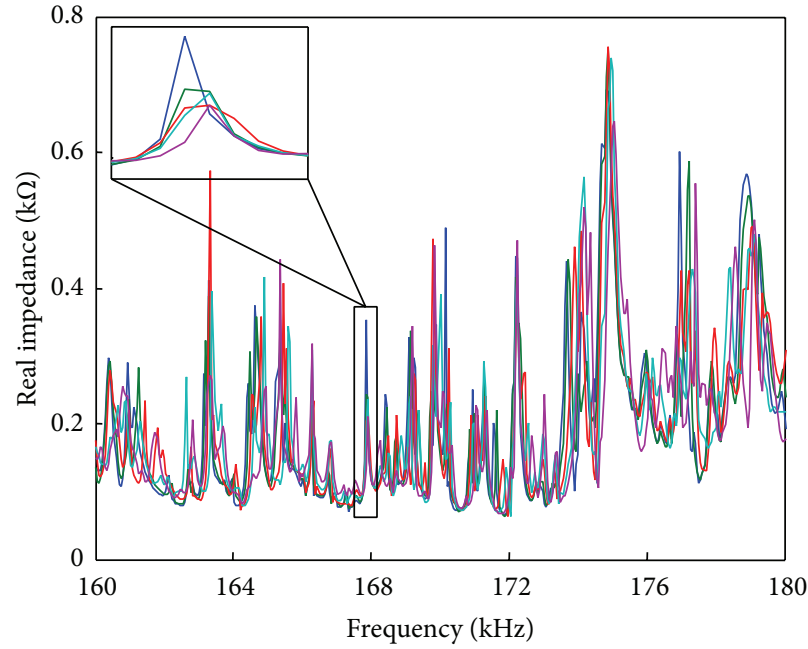

(b)

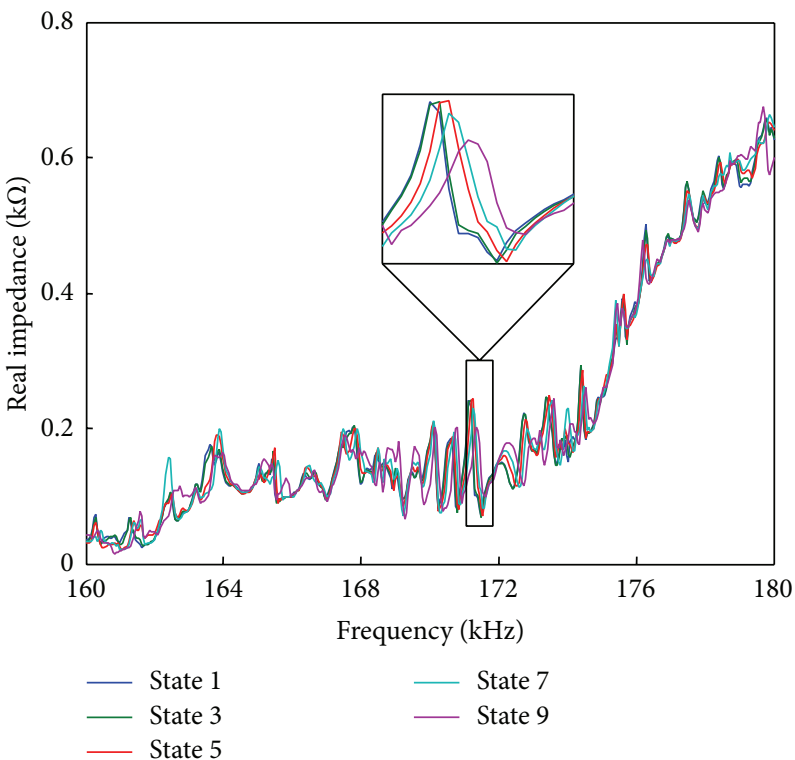

(d)

FIGURE 5: Impedance spectra measured from different rods under some certain states and zoomed parts: (a) rod 1; (b) rod 2; (c) rod 3; (d) $\operatorname{rod} 4$.

to rod 2 are generally larger than those corresponding to rod 1 and that RMSD values corresponding to rod 3 are larger than those corresponding to rod 4, indicating that the variations of impedance spectra are different, even at the same joint. However, for one certain rod, RMSD values increase monotonously. Therefore, damage location can be determined by the differences among RMSD values corresponding to different rods and the severity of loosened rod can be evaluated based on the characteristics of monotonic increment of RMSD values.

\subsection{Loosening Identification and Quantification Based on BP} Neural Network. In this study, one BP neural network was adopted to process measured data so that loosening levels could be expressed quantitatively and more clearly. Figure 7 provides a simplified model of this kind of network. Training samples and testing samples are imported from input layer. The calculated results were exported from output layer.

Theoretically, neural network with one hidden layer is enough to simulate any nonlinear relationship between inputs and outputs. But this network could not meet the requirements of too high precision. Therefore, in this study, neural network included one input layer, two hidden layers, and one output layer. Sigmoid nonlinear function was selected as transfer function. Connective weights and bias values were assigned randomly and network was partly initialized.

Each impedance spectrum measured by the impedance analyzer contains 401 data points. If these data points are all treated as input vectors, network training speed will become 


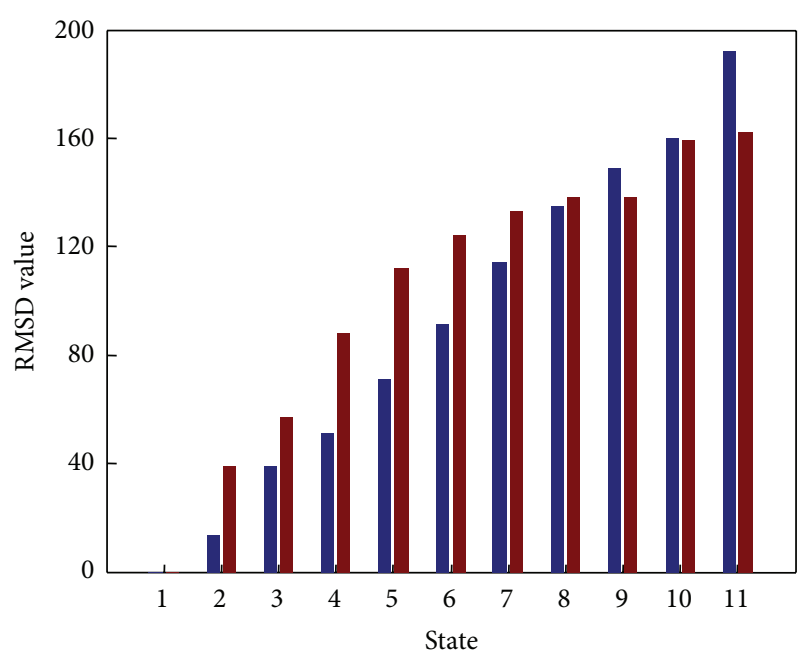

Variation of rod 1

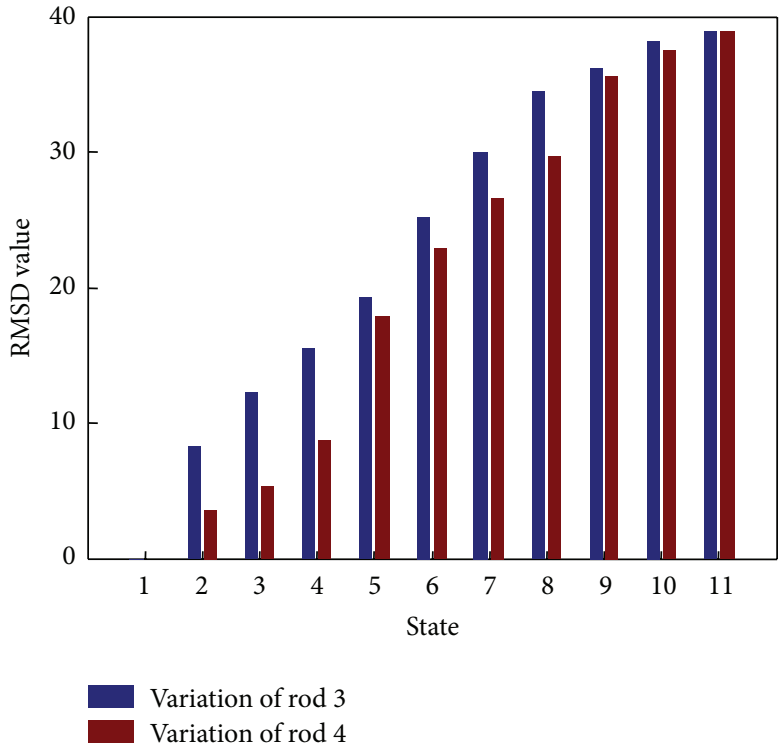

(b)

FIGURE 6: RMSD values corresponding to the rods connected to the same joints under different states: (a) rod 1 and $\operatorname{rod} 2$; (b) rod 3 and rod 4.

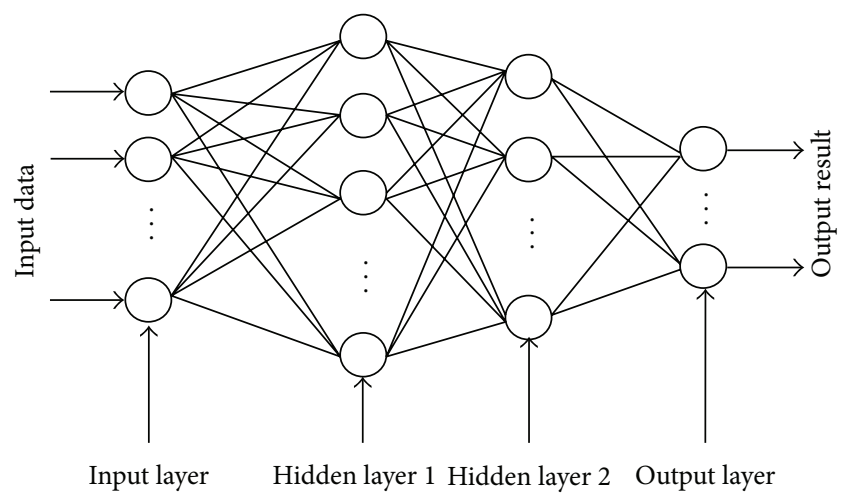

FIGURE 7: Simplified model of BP neural network.

too slow. And it is also too hard to achieve error limit. Thus, data points will always be compressed under this state. Then the feature of structural damage can be represented by a small number of data points. However, some information will be lost during the data compression process. After compressing, if there are less data points, more information will be lost, indicating that the selected number of data points is very important.

As shown in Figure 6, RMSD values calculated from the whole frequency band increased as the rods were restored to their initial positions. However, as shown in Figures 5(a) and 5(b), impedance spectra also show monotonous variation in some local frequency bands. Even if the total frequency range is divided into several subbands and RMSD values are calculated, damage features can be distinguished from each other. RMSD values calculated from different subbands are shown in Figure 8. The total frequency range was divided into 10 subbands. For the rods 1 to 4 , state 1 was treated as their respective baselines and (2) was applied to calculate RMSD values. In order to ensure that all subfrequencies contained the same number of data points, the first frequency band of $160-162 \mathrm{kHz}$ included points 1 to 41 and the second frequency band of $162-164 \mathrm{kHz}$ included points 41 to 81 . A series of numbers were assigned to represent different frequency bands. For example, the numbers 1 and 2 represent $160-162 \mathrm{kHz}$ and $162-164 \mathrm{kHz}$, respectively.

As illustrated in Figure 8(a), RMSD values increase monotonically in $160-162 \mathrm{kHz}$ and $170-180 \mathrm{kHz}$. Furthermore, the same phenomenon appears in the following frequency bands: $166-172 \mathrm{kHz}$ and $174-178 \mathrm{kHz}$ (Figure 8(b)); $162-166 \mathrm{kHz}, 170-174 \mathrm{kHz}$, and $178-180 \mathrm{kHz}$ (Figure 8(c)); $168-178 \mathrm{kHz}$ in Figure 8(d). And it can be found that, in other frequency bands, these RMSD values do not increase monotonically. But experimental results by Min proved that neural network could adjust the weights as required during the training process $[21,22]$. If the variation trend of RMSD values of one band is similar to that of target results, RMSD values measured in this band have the most significant influence on output results after the training, such as band 1, band 6, and band 8 in Figure 8(a). But the influences on other bands are small. Therefore the compressed impedance data could be all treated as input vectors and the effect of irregular changes will decrease as training error converges to target value.

It is obvious that RMSD values of different subfrequency ranges corresponding to the variations of joints $\mathrm{A}$ and $\mathrm{B}$ are different. And those values corresponding to the variations of rods connected to the same joint are not the same as well. RMSD values calculated from the variations of rods connected to joint $\mathrm{A}$ are always larger than the RMSD values 


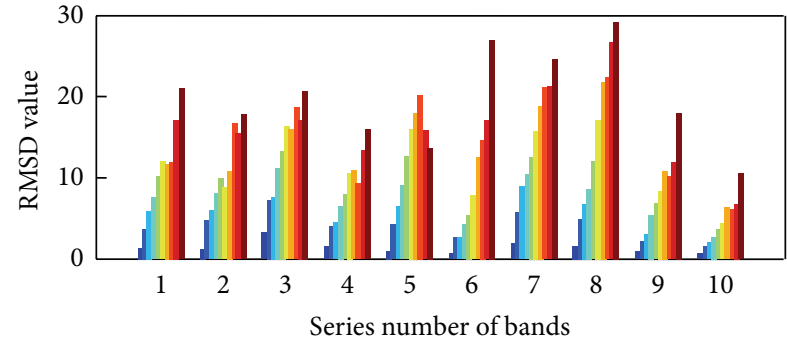

(a)

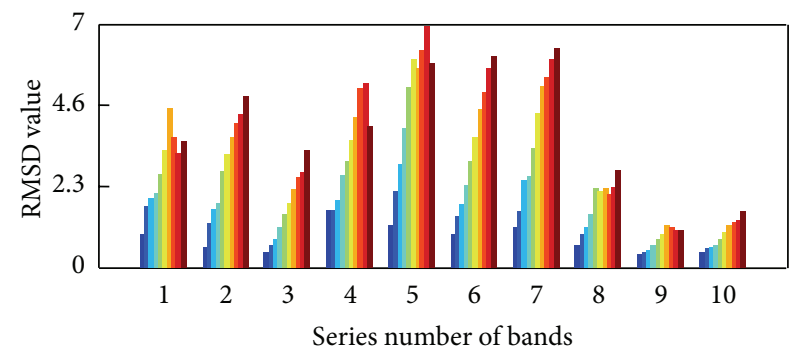

State 1
State 2
State 3
State 4

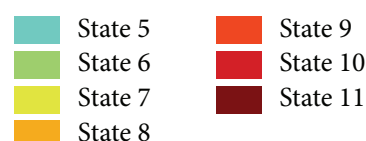

(c)

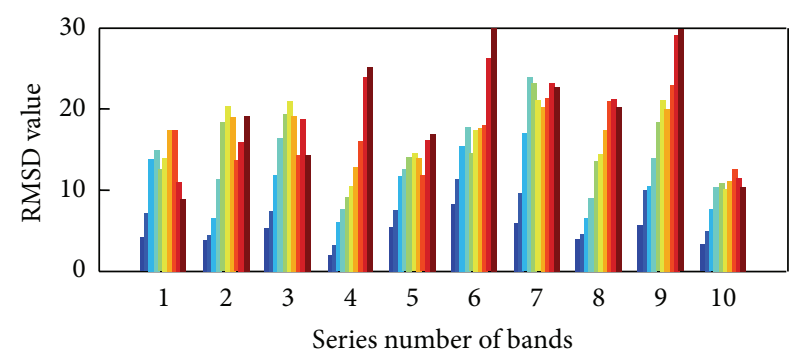

(b)

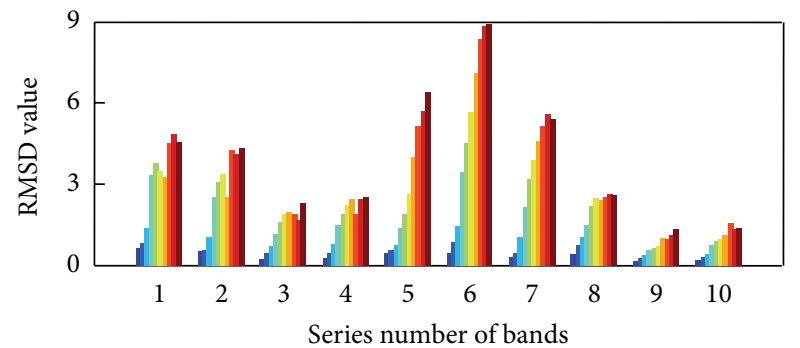

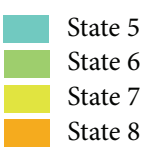

(d)

FiguRE 8: RMSD values of different subfrequency ranges for the structure under different health states and the mapping between different health states and the color of histograms: (a) the variation of rod 1; (b) the variation of rod 2; (c) the variation of rod 3; (d) the variation of $\operatorname{rod} 4$.

of rods connected to joint $\mathrm{B}$, as indicated above. Larger RMSD values corresponding to rod 1 largely fall in the $170-176 \mathrm{kHz}$ range and RMSD values corresponding to rod 2 fall in $166-168 \mathrm{kHz}, 170-172 \mathrm{kHz}$, and $176-178 \mathrm{kHz}$. For rods 3 and 4 , larger RMSD values appear in the same range, $168-170 \mathrm{kHz}$, $170-172 \mathrm{kHz}$, and $172-174 \mathrm{kHz}$. However, unlike RMSD values for rod 3, RMSD values for rod 4 in $170-172 \mathrm{kHz}$ are larger than those in the other two frequency ranges. In conclusion, RMSD values can be calculated after the whole test frequency range was divided into 10 subbands. Damage information can be represented by 10 data points instead of 401 data points. And these compressed data points also contain different damage features.

However, some information will be lost after data compression. In Figures 6(a) and 6(b), when rods 1 and 2 are both under state 10, RMSD values are almost the same. But in Figure 8 , when rod 1 is under state 10 , the maximum RMSD value appears in $174-176 \mathrm{kHz}$ and the maximum RMSD value of rod 2 appears in $176-178 \mathrm{kHz}$. Their amplitudes are 26.68 and 24.25, respectively. According to the comparison of these figures, if there are less compressed data points, more damage information will be lost. For example, different damage conditions shown in Figure 8 had 10 data points while Figure 6 had only 1 data point. Hence, it is hard to distinguish different damage states.

In this study, RMSD values are normalized through (3) and treated as input vectors. Within these vectors, 12 data sets were selected as testing samples, while others were treated as training samples:

$$
R=\frac{r-r_{\min }}{r_{\max }-r_{\min }}
$$

In this equation, $r$ and $R$ represent RMSD values before and after normalization, respectively. $r_{\min }$ and $r_{\max }$ represent the minimum and maximum RMSD values in all input data, respectively. From the previous discussion, it can be concluded that the larger the number of the divided frequency subbands is, the more the comprehensive information compressed data will be contained and the easier it is to improve the ability of damage identification. Since more input data would decrease training rate and lead to the difficulty of network convergence, more tests were performed to select the properly divided frequency subbands. Finally, the number of divided frequency subbands was confirmed to be 25 . The first hidden layer contained 100 neurons. The second hidden layer contained 50 neurons and output layer contained 3 neurons. The first and the second neurons of output layer were defined as damage location and the third neurons were defined as damage information. The three neurons were, respectively, joint number, rod number, and severity. Joint number 0 represents joint $\mathrm{A}$ while joint number 1 represents joint $\mathrm{B}$. Rod number 0 represents rod 1 or rod 3 while rod number 1 represents rod 2 or rod 4 . Severity outputs were $0,0.1,0.2$, $0.3,0.4,0.5,0.6,0.7,0.8,0.9$, and 1 , which were multiplied by 10 to obtain intuitive results. For example, 012 indicated that 
TABLE 1: Expected outputs and actual outputs of testing samples.

\begin{tabular}{|c|c|c|c|c|c|c|c|c|c|c|c|}
\hline \multirow{3}{*}{ Rod 1} & \multicolumn{2}{|c|}{ Damage states } & \multicolumn{3}{|c|}{2} & \multicolumn{3}{|c|}{4} & \multicolumn{3}{|c|}{8} \\
\hline & Output & Expected & 0 & 0 & $1-3$ & 0 & 0 & $3-5$ & 0 & 0 & $7-9$ \\
\hline & citput & Actual & 0.0004 & 0.0001 & 1.6398 & 0.0004 & 0.0007 & 3.8930 & 0.0023 & 0.0659 & 7.3636 \\
\hline \multirow{3}{*}{ Rod 2} & \multicolumn{2}{|c|}{ Damage states } & & 1 & & & 3 & & & 7 & \\
\hline & \multirow{2}{*}{ Output } & Expected & 0 & 1 & $0-2$ & 0 & 1 & $2-4$ & 0 & 1 & $6-8$ \\
\hline & & Actual & 0.0062 & 0.9990 & 1.0841 & 0.0061 & 0.9858 & 2.3948 & 0.0031 & 0.9771 & 6.5686 \\
\hline \multirow{3}{*}{ Rod 3} & \multicolumn{2}{|c|}{ Damage states } & & 2 & & & 7 & & & 9 & \\
\hline & \multirow{2}{*}{ Output } & Expected & 1 & 0 & $1-3$ & 1 & 0 & $6-8$ & 1 & 0 & $8-10$ \\
\hline & & Actual & 0.9977 & 0.0002 & 1.9314 & 0.9959 & 0.0085 & 8.0894 & 0.9956 & 0.0456 & 8.8745 \\
\hline \multirow{3}{*}{ Rod 4} & \multicolumn{2}{|c|}{ Damage states } & & 1 & & & 5 & & & 8 & \\
\hline & \multirow{2}{*}{ Output } & Expected & 1 & 1 & $0-2$ & 1 & 1 & $4-6$ & 1 & 1 & $7-9$ \\
\hline & & Actual & 0.9946 & 0.9975 & 1.6656 & 0.9960 & 0.9982 & 5.2482 & 0.9960 & 0.9173 & 8.5058 \\
\hline
\end{tabular}

rod 2 connected to joint A was under damage state 2; 113 indicated that rod 4 connected to joint $\mathrm{B}$ was under damage state 3.

For rod 1, damage states 2, 4, and 8 were selected as testing samples, as shown in Table 1. For other rods, testing samples were also shown in Table 1 . Then other data sets were treated as training samples. The target error was 0.0005 and neural network was built and trained. After training for 4634 times, target error reached the allowed limits. Testing samples were imported into the trained neural network and testing results were shown in Table 1 . However, if one sample was not involved in training process, the network could only evaluate its damage state through the information contained in the training sample. For test samples, only the upper and lower limits of damage severity were given. Since the increment of adjacent RMSD values is irregular, the trained network can determine the range of damage severity and cannot yield an exact value. For one sample corresponding to damage state $n$, if its damage severity is classified into the level between $n-1$ and $n+1$, this result can be treated as correct result. According to this criterion, damage location can be identified solely by the trained network and almost all damage states can be evaluated correctly. Only the output result corresponding to rod 3 under state 7 has an error rate which is $1.1 \%$ above the upper limit. This means that the trained network cannot only recognize the location of loose rods but also evaluate the loosening levels.

\section{Conclusions}

EMI technique and artificial neural network technique were combined together to evaluate the health state of truss structures. Firstly, the measured data were greatly compressed through calculating the RMSD values of different frequency ranges, respectively. The results showed that compressed data contained damage features under different states and that the quantity of the remaining information was positively correlated to the number of compressed data points. In this way, the training rate of the network can be improved and remaining information is enough to build and train one BP neural network. Testing results showed that trained neural network could not only recognize the location of loose rods but also quantitatively evaluate its loosening levels. Therefore, the combination of EMI technique and artificial neural network will have a broad application prospect in intelligent health monitoring of complex structures including truss structures.

\section{Conflict of Interests}

The authors declare that there is no conflict of interests regarding the publication of this paper.

\section{Acknowledgments}

The work was supported by the National Natural Science Foundation of China (nos. 51235001, 11272021, and 50975006), Beijing Natural Science Foundation (no. 1122007), General Program of Science and Technology Development Project of Beijing Municipal Commission of Education (no. KM201010005003), the Importation and Development of High-Caliber Talents Project of Beijing Municipal Institutions (CIT\&TCD201304048), and Beijing Nova Program (no. 2008A015).

\section{References}

[1] Y. Y. Lim, S. Bhalla, and C. K. Soh, "Structural identification and damage diagnosis using self-sensing piezo-impedance transducers," Smart Materials \& Structures, vol. 15, no. 4, pp. 987-995, 2006.

[2] V. Giurgiutiu, K. Harries, M. Petrou, J. Bost, and J. B. Quattlebaum, "Disbond detection with piezoelectric wafer active sensors in RC structures strengthened with FRP composite overlays," Earthquake Engineering and Engineering Vibration, vol. 2, no. 2, pp. 213-223, 2003.

[3] H. A. Sodano, G. Park, and D. J. Inman, "An investigation into the performance of macro-fiber composites for sensing and structural vibration applications," Mechanical Systems and Signal Processing, vol. 18, no. 3, pp. 683-697, 2004.

[4] F. P. Sun, Z. Chaudhry, C. Liang, and C. A. Rogers, "Truss structure integrity identification using PZT sensor-actuator," Journal of Intelligent Material Systems and Structures, vol. 6, no. 1, pp. 134-139, 1995. 
[5] S. Ritdumrongkul and Y. Fujino, "Identification of the location and level of damage in multiple-bolted-joint structures by PZT actuator-sensors," Journal of Structural Engineering, vol. 132, no. 2, pp. 304-311, 2006.

[6] L. V. Palomino, K. M. Tsuruta, J. R. V. Mour Jr., D. A. Radea, V. Steffen Jr., and D. J. Inman, "Evaluation of the influence of sensor geometry and physical parameters on impedance-based structural health monitoring," Shock and Vibration, vol. 19, no. 5, pp. 811-823, 2012.

[7] W. Yan, C. W. Lim, J. B. Cai, and W. Q. Chen, "An electromechanical impedance approach for quantitative damage detection in Timoshenko beams with piezoelectric patches," Smart Materials and Structures, vol. 16, no. 4, pp. 1390-1400, 2007.

[8] Y. Yang and B. S. Divsholi, "Sub-frequency interval approach in electromechanical impedance technique for concrete structure health monitoring," Sensors, vol. 10, no. 12, pp. 11644-11661, 2010.

[9] B. S. Divsholi and Y. Yang, "Health monitoring of steel structures using sub-frequency electromechanical impedance technique," Journal of Nondestructive Evaluation, vol. 31, no. 3, pp. 197-207, 2012.

[10] V. G. M. Annamdas and Y. Yang, "Practical implementation of piezo-impedance sensors in monitoring of excavation support structures," Structural Control \& Health Monitoring, vol. 19, no. 2, pp. 231-245, 2012.

[11] R. Shanker, S. Bhalla, and A. Gupta, "Integration of electromechanical impedance and global dynamic techniques for improved structural health monitoring," Journal of Intelligent Material Systems and Structures, vol. 21, no. 3, pp. 285-295, 2010.

[12] V. Giurgiutiu and A. Zagrai, "Damage detection in thin plates and aerospace structures with the electro-mechanical impedance method," Structural Health Monitoring, vol. 4, no. 2, pp. 99-118, 2005.

[13] V. Lopes Jr., G. Park, H. H. Cudney, and D. J. Inman, "Impedance-based structural health monitoring with artificial neural networks," Journal of Intelligent Material Systems and Structures, vol. 11, no. 3, pp. 206-214, 2000.

[14] C. Liang, F. P. Sun, and C. A. Rogers, "Coupled electromechanical analysis of adaptive material systemsdetermination of the actuator power consumption and system energy transfer," Journal of Intelligent Material Systems and Structures, vol. 5, no. 1, pp. 12-20, 1997.

[15] S. Na and H. K. Lee, "Resonant frequency range utilized electro-mechanical impedance method for damage detection performance enhancement on composite structures," Composite Structures, vol. 94, no. 8, pp. 2383-2389, 2012.

[16] S. Na and H. K. Lee, "A technique for improving the damage detection ability of the electro-mechanical impedance method on concrete structures," Smart Materials and Structures, vol. 21, no. 8, Article ID 085024, 2012.

[17] G. Park, H. Sohn, C. R. Farrar, and D. J. Inman, "Overview of piezoelectric impedance-based health monitoring and path forward," Shock and Vibration Digest, vol. 35, no. 6, pp. 451-463, 2003.

[18] S. Park, J. Lee, C. Yun, and D. J. Inman, "A built-in active sensing system-based structural health monitoring technique using statistical pattern recognition," Journal of Mechanical Science and Technology, vol. 21, no. 6, pp. 896-902, 2007.

[19] J. L. Rickli and J. A. Camelio, "Damage detection in assembly fixtures using non-destructive electromechanical impedance sensors and multivariate statistics," International Journal of Advanced Manufacturing Technology, vol. 42, no. 9-10, pp. 10051015, 2009.

[20] A. Hai, A. Weiguang, Y. Duohe, and W. Binsheng, "Static force reliability analysis of truss structure with piezoelectric patches affixed to its surface," Chinese Journal of Aeronautics, vol. 22, no. 1, pp. 22-31, 2009.

[21] J. Min, S. Park, and C. Yun, "Impedance-based structural health monitoring using neural networks for autonomous frequency range selection," Smart Materials and Structures, vol. 19, no. 12, Article ID 125011, 2010.

[22] J. Min, S. Park, C. Yun, C. Lee, and C. Lee, "Impedance-based structural health monitoring incorporating neural network technique for identification of damage type and severity," Engineering Structures, vol. 39, pp. 210-220, 2012. 

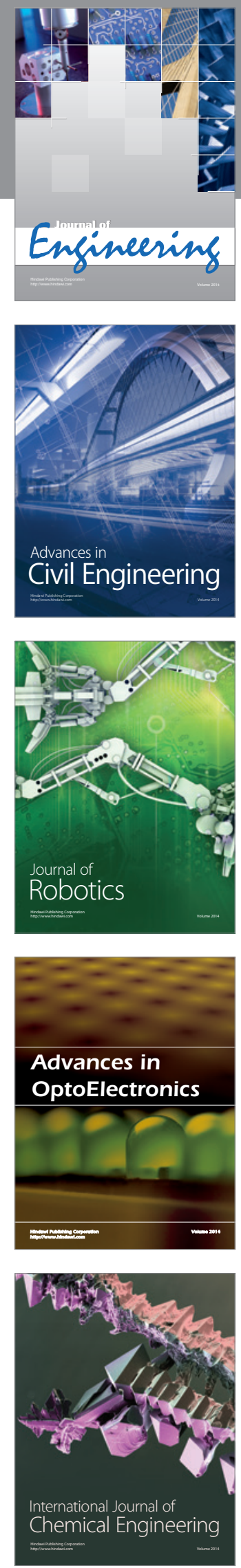

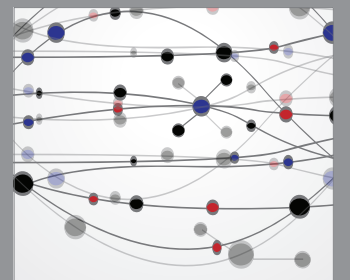

The Scientific World Journal
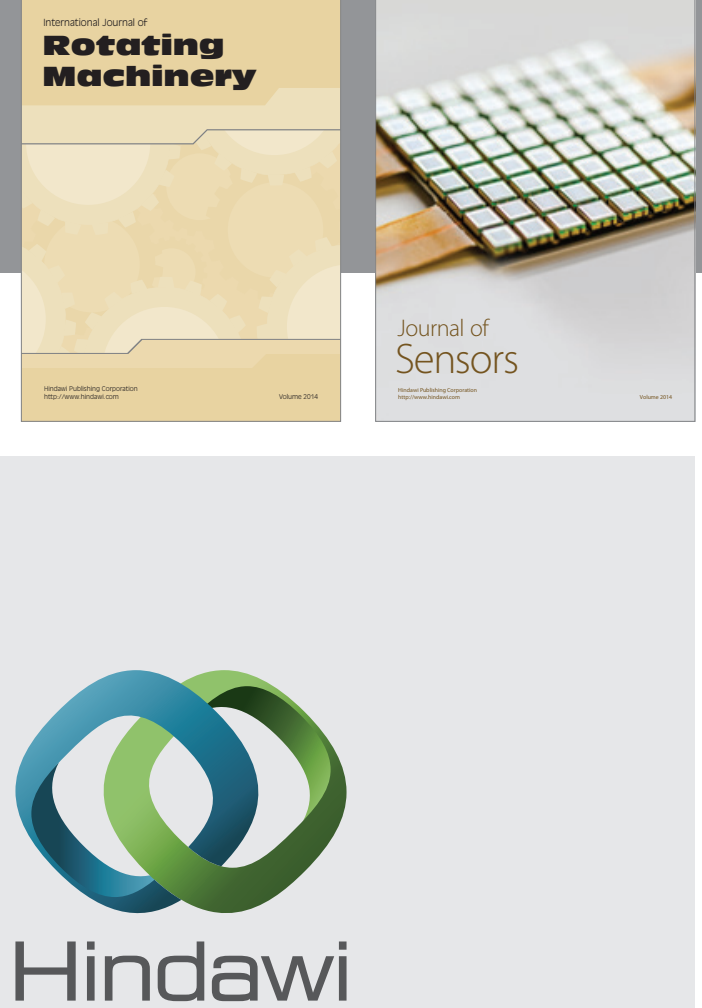

Submit your manuscripts at http://www.hindawi.com
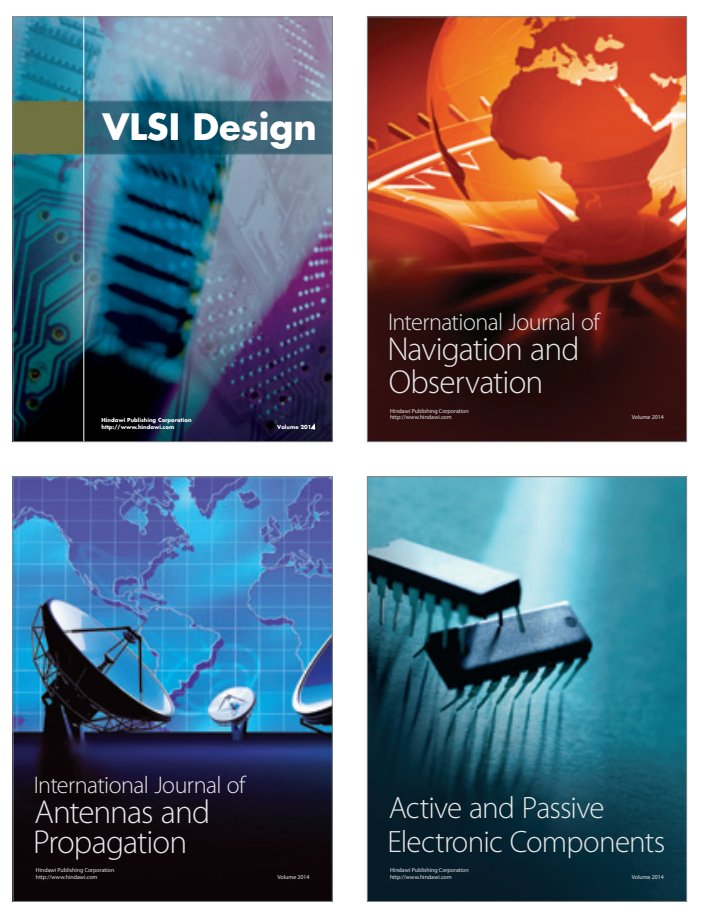
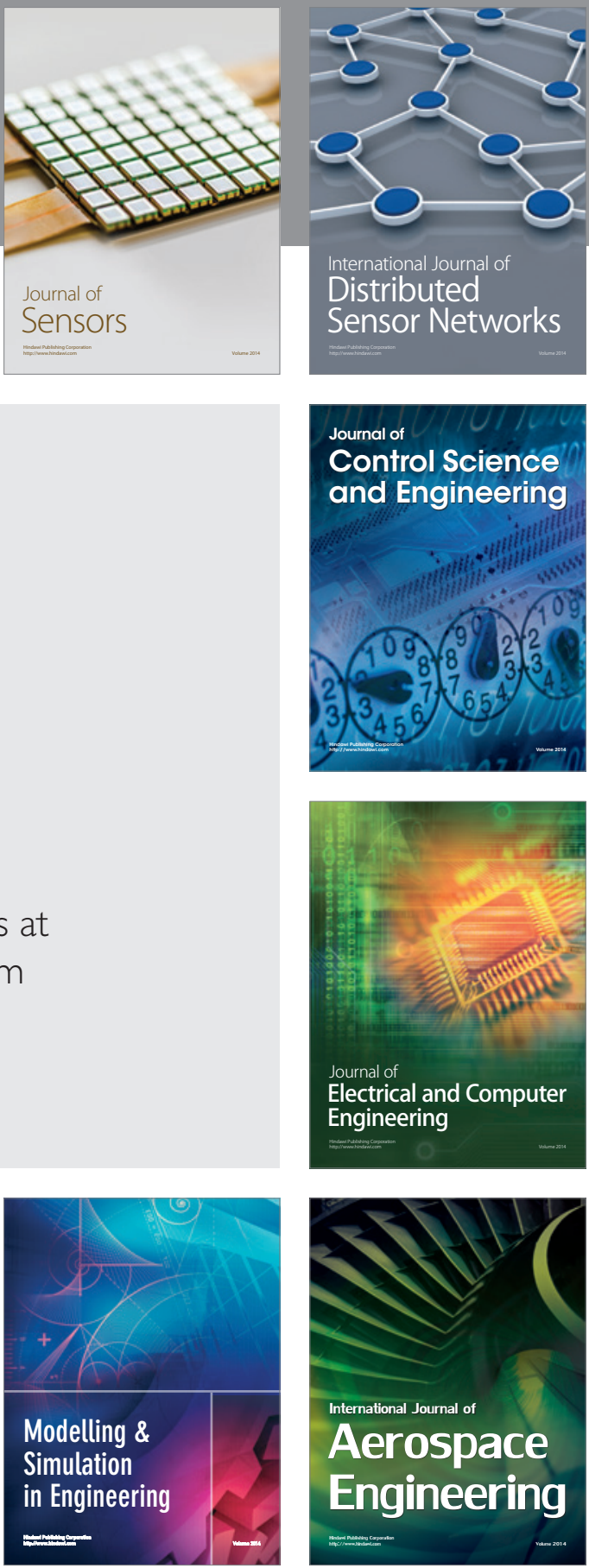

Journal of

Control Science

and Engineering
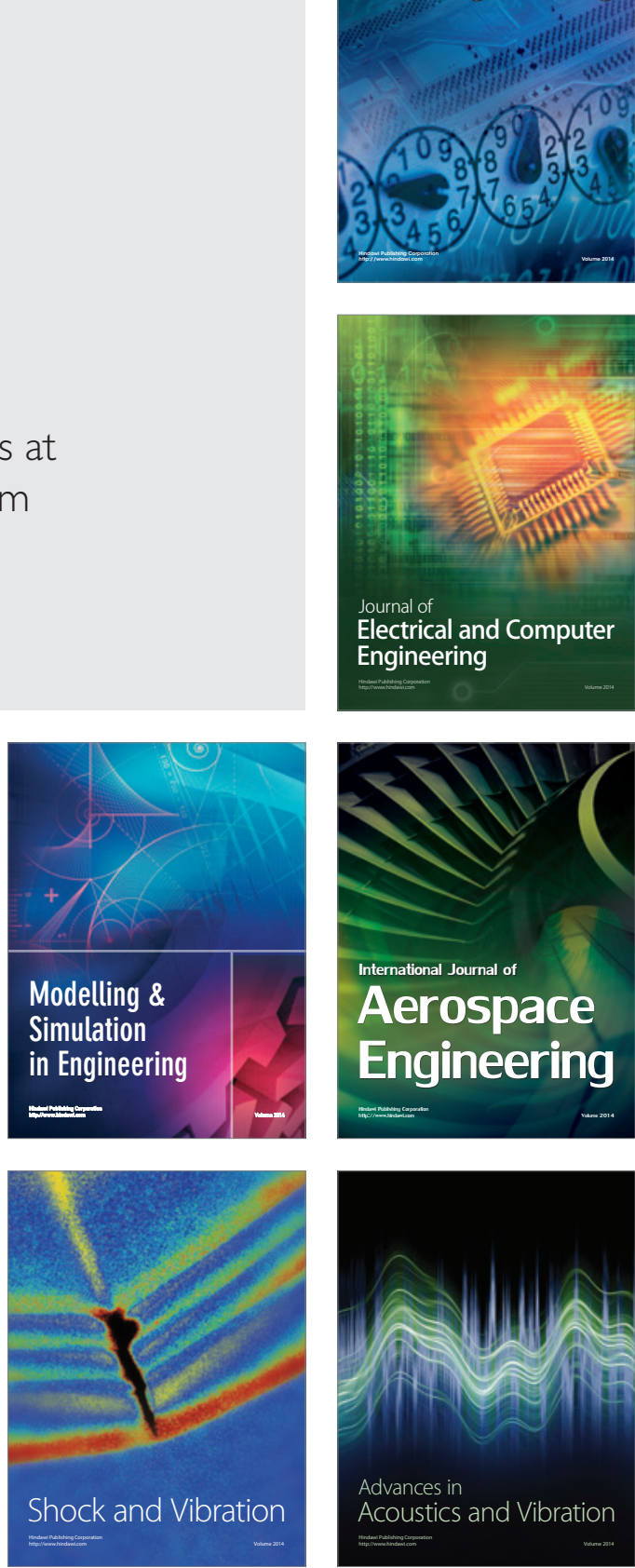